\title{
A CASE OF DISSEMINATED HISTOPLASMOSIS IN AN IMMUNOCOMPETENT PERSON
}

Debkumar Ray', Debashis Roy Burman², Indranil Dhar ${ }^{3}$

\section{HOW TO CITE THIS ARTICLE:}

Debkumar Ray, Debashis Roy Burman, Indranil Dhar. "A Case of Disseminated Histoplasmosis in an Immunocompetent Person". Journal of Evolution of Medical and Dental Sciences 2014; Vol. 3, Issue 24, June 16; Page: 6601-6604, DOI: $10.14260 /$ jemds/2014/2787

ABSTRACT: Histoplasmosis is a fungal disease caused by Histoplasma capsulatum. Most infections are asymptomatic or self-limiting but some may develop acute pulmonary infections or severe and progressive disseminated infection. Disseminated histoplasmosis frequently affects the adrenal gland; however, unilateral involvement in immunosuppressed patients is the usual presentation. We report a case of a middle aged immunocompetent male with history of weight loss, fever, hepatomegaly and bilateral adrenal mass who was diagnosed to be suffering from disseminated histoplasmosis and successfully treated with Itraconazole.

KEYWORDS: Histoplasma capsulatum, Adrenal mass, Itraconazole.

INTRODUCTION: Histoplasmosis is an infectious disease caused by the dimorphic fungus Histoplasma capsulatum. Histoplasmosis is common in AIDS patients but it is relatively rare in nonimmunocompromised person in India. The infection is self-limiting and restricted to lungs in 99\% of individuals with no pre-existing immunological defects. The remaining $1 \%$, however progress to either disseminated or chronic disease involving lungs, liver, spleen, lymph node, bone marrow, skin, mucous membrane and adrenal ${ }^{1}$.The clinical presentation of the disseminated disease includes pyrexia, anorexia, nausea, vomiting, weight loss and fatigue. These symptoms are nonspecific and resemble other chronic infections and malignancies; so high index of clinical suspicion, recognition of the common modes of presentation and excellent laboratory support is needed for its diagnosis.

THE CASE: A 48 years old non diabetic male presented to us, with low grade continuous fever with weight loss for last six months. Fever was associated with occasional mild dry cough, marked anorexia and generalized weakness. It was not associated with chills or rigors, night sweat or evening rise of temperature. There was gradual darkening of whole body which was more prominent over face, upper limbs and upper part of the trunk. During this period he received several courses of different antibiotics and was on empirical anti-tubercular therapy for 20days without any improvement.

On physical examination only hepatomegaly was present. Disseminated tuberculosis, malignancies were considered as differential diagnosis. His complete blood count showed mild anemia with microcytic normochromic pattern [value- haemoglobin- 9.8gm/dl, MCV- $76 \mathrm{fL}$ ]. Erythrocyte sedimentation rate was found to be increased [value- $130 \mathrm{~mm}$ at the end of $1^{\text {st }}$ hour]. Total white blood cell count was within normal limit but relative lymphocytosis was present [Hb12.6, TLC- 5900: N69, L29, E1, M1, ESR-29].

Liver function test showed hypoalbuminemia and slightly elevated liver enzymes [T bilirubin0.9, T. protein- 8 gm/dl, SGOT- 64 IU/L, SGPT- 54 IU/L, alkaline phosphatase- 138]. Blood sugar (fasting \& post prandial) was within normal limit. Blood culture did not show any growth. He was 
nonreactive for HIV-ELISA and rK39 (for visceral leishmaniasis). Chest X-ray was normal. Ultrasonography of abdomen showed hepatomegaly with well-defined bilateral hypoechoic adrenal space occupying lesion.

CECT of abdomen showed bilateral adrenal mass measuring $37 \mathrm{~mm} \times 42 \mathrm{~mm} \times 34 \mathrm{~mm}$ in right and $37 \mathrm{~mm} \times 23 \mathrm{~mm} \times 18 \mathrm{~mm}$ in left (picture 1). CT guided FNAC was done and cytological examination showed presence of plenty of intracellular and extracellular budding yeast cells (picture 2). Morning fasting serum cortisol at 8 am was within normal limit [value- $11.50 \mathrm{mcg} / \mathrm{dl}$ (Ref-3.7-19.4mcg/dl)] and serum ACTH level was also normal [value-58pg/ml (Ref- $6.0-76 \mathrm{pg} / \mathrm{ml}$ )]. Then the patient was put on itraconazole $200 \mathrm{mg}$ thrice daily as a loading dose followed by $200 \mathrm{mg}$ twice daily. Later the fungal culture from the aspirate showed brownish white colony of histoplasma.

Within 7 days of treatment his fever subsided and after 1 month of treatment with itraconazole he started to gain weight with increased appetite. Itraconazole concentration in blood could not be measured due to unavailability of the test facility in our institute. After 10 months of treatment with itraconazole patient was asymptomatic with regression in adrenal mass size on follow up ultrasonography and Itraconazole was extended for another two months.

DISCUSSION: Disseminated histoplasmosis is sporadically reported disease in India. In overview of histoplasmosis reported in India from 1968 to 1992, Padhye et al. listed 25 cases as authentically diagnosed $^{2}$. He concluded histoplasmosis is India has mainly extra pulmonary manifestations and in the majority it involved oral cavity. Subramanian S et al has published a retrospective analysis of 19 patients with disseminated histoplasmosis from 1988 to 1999 and concluded diabetes mellitus and HIV as a major comorbid condition. ${ }^{3}$ Disseminated histoplasmosis may affect almost all systems, including the reticuloendothelial system, lungs, gastrointestinal tract, renal tract, central nervous system, bone marrow, adrenal glands and skin. ${ }^{4}$

Adrenal involvement is common (60 to 90\%) but overt adrenal insufficiency is found in fewer than 10 per cent of cases ${ }^{4}$. Histopathology using stains for fungi and cultures, antigen detection, and/or serologic tests for Histoplasma-specific antibodies may be employed to diagnose histoplasmosis. ${ }^{5}$ But the serological tests are not available readily in resource limited areas. Cytological examination shows that the intracellular forms are situated within the cytoplasm of histiocytes, where they appear as numerous small spherical or oval yeast forms surrounded by a clear ring of space that resembles a capsule.

Lipid formulation of amphotericin B (3.0-5.0 mg/kg daily intravenously for 1-2 weeks) followed by itraconazole ( $200 \mathrm{mg} 3$ times daily for 3 days and then $200 \mathrm{mg}$ twice daily, for a total of at least 12 months) is recommended for severe and moderately severe disease. ${ }^{6}$ The deoxycholate formulation of amphotericin B (0.7-1.0 mg/kg daily intravenously) is an alternative to a lipid formulation in patients who are at a low risk for nephrotoxicity. ${ }^{6}$ For mild to moderate disease only itraconazole is used. In the above mentioned case no sign of severity was present so itraconazole was used.

This case emphasizes the fact that adrenal histoplasmosis may occur in immunocompetent patients and has to be considered in the differential diagnosis of prolonged fever, weight loss, hepatomegaly and adrenal masses. Adrenal insufficiency has to be monitored and antifungal therapy should be maintained for at least one year. 


\section{CASE REPORT}

\section{REFERENCES:}

1. Tewari RP, Wheat LJ, Ajello L, Agents of histoplasmosis. In: Ajello L, Hay J, editors. Topley and Wisons Microbiology and Microbial infections. 9th edition, volume4, Arnold: London; 1998; p.373-93.

2. Padhyae AA, Pathak AA, Katyar VJ, Hazare VK, Kaufman L. Oral histoplasmosis in India: Case report and overview of the cases reported during 1968-1992. J Med Vet Mycol 1994; 32: 93103.

3. Subramanian S, Abraham OC, Rupali P, Zachariah A, Mathews MS, Mathai D. Disseminated histoplasmosis. J Assoc Physicians India 2005; 53: 185-9.

4. Goodwin, RA Jr, Shapiro, JL, Thurman, GH, et al. Disseminated histoplasmosis: Clinical and pathologic correlations. Medicine (Baltimore) 1980; 59: 1.

5. Wheat, LJ. Approach to the diagnosis of the endemic mycoses. Clin Chest Med 2009; 30: 379.

6. L. Joseph Wheat1, Alison G. Freifeld3 et.al. Clinical Practice Guidelines for the Management of Patients with Histoplasmosis: 2007; Update by the Infectious Diseases Society of America.

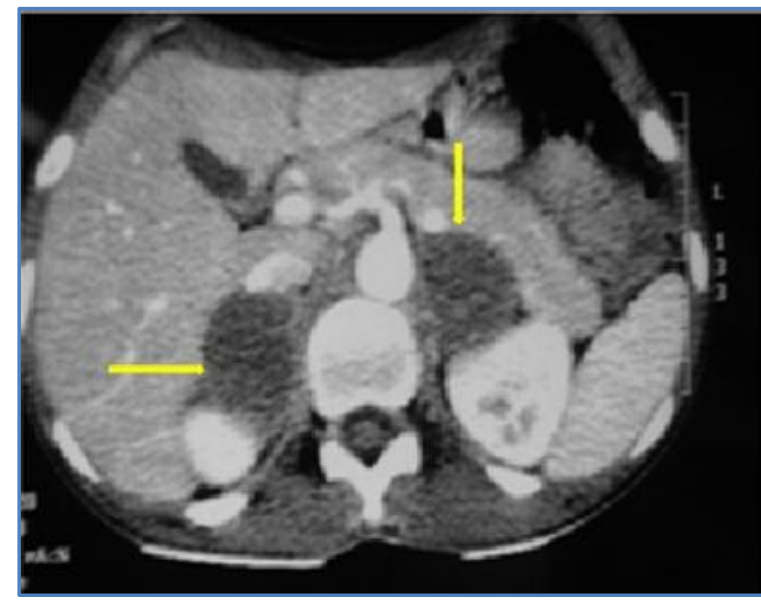

\section{Picture 1: Bilateral hypodense adrenal mass}

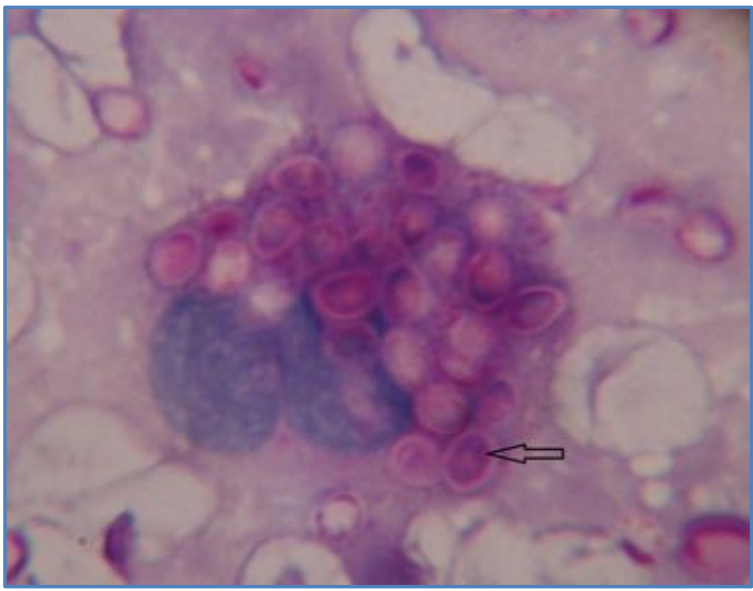

Picture 2: Capsulated Yeast form of Histoplasma within macrophage 


\section{CASE REPORT}

\section{AUTHORS:}

1. Debkumar Ray

2. Debashis Roy Burman

3. Indranil Dhar

\section{PARTICULARS OF CONTRIBUTORS:}

1. Assistant Professor, Department of Biochemistry, B. S. Medical College, Bankura, W. B.

2. Assistant Professor, Department of Laboratory Oncology, (Oncopathlolgy), Medical College, Kolkata.

3. Assistant Professor, Department of Laboratory Medicine, School of Tropical Medicine, Kolkata.

\section{NAME ADDRESS EMAIL ID OF THE} CORRESPONDING AUTHOR:

Dr. Debkumar Ray,

KL-1, Ganga Yamuna Apartments, Block-B, Flat: C\&D, Aswininagar, Kolkata-700059.

Email: dr.debkumar@gmail.com

Date of Submission: 25/05/2014. Date of Peer Review: 26/05/2014. Date of Acceptance: 04/06/2014. Date of Publishing: 10/06/2014. 